\title{
Koronarangiografie bei Nephropathie: Besser ohne RAS-Blocker
}

\author{
ACE-Hemmer und $\mathrm{AT}_{1}$-Rezeptor-Blocker (RAS-Blocker) führen bei \\ Patienten mit Niereninsuffizienz nach Kontrastmittelgabe zu einem \\ Kreatininanstieg und sollten deshalb mindestens 24 Stunden vor \\ einer Herzkatheteruntersuchung abgesetzt werden.
}

n die Pilotstudie wurden 208 Patienten mit leichter bis schwerer Niereninsuffizienz (Serumkreatinin über $1,5 \mathrm{mg} / \mathrm{ml}$ bzw. GFR nicht unter $15 \mathrm{ml} / \mathrm{min}$ ) und einer Therapie mit ACE-Hemmern $(72,1 \%)$ oder $\mathrm{AT}_{1}$-Rezeptor-Blockern $(27,9 \%)$ aufgenommen. Nach Zufallskriterien wurde diese Therapie entweder weitergeführt $(\mathrm{n}=102)$ oder mindestens 24 Stunden vor bis 48-96 Stunden nach der Herzkatheteruntersuchung abgesetzt $(\mathrm{n}=106)$. Alle Patienten wurden gut hydriert.

Die Ergebnisse (Tab. 1) zeigen einerseits, dass ACE-Hemmer und $\mathrm{AT}_{1}$-Rezeptor-Blocker die Nierenfunktion verschlechtern und klinische Komplikationen begünstigen können, wenn sie während einer Herzkatheteruntersuchung mit Kontrastmitteln weiter verordnet werden. Andererseits gehen nach dem Absetzen der RAS-Blocker erwünschte

\begin{tabular}{|c|c|c|c|}
\hline & $\begin{array}{l}\text { Mit RAS- } \\
\text { Blockern }\end{array}$ & $\begin{array}{l}\text { Ohne } \\
\text { RAS- } \\
\text { Blocker }\end{array}$ & $p$-Wert \\
\hline $\begin{array}{l}\text { Kreatinin- } \\
\text { anstieg } \\
\text { (mg/dl) }\end{array}$ & 0,3 & 0,1 & 0,03 \\
\hline $\begin{array}{l}\text { Anstieg des } \\
\text { Kreatinins } \\
\geq 0,5 \mathrm{mg} / \mathrm{dl} \\
\text { oder }>25 \%\end{array}$ & $18,4 \%$ & $10,9 \%$ & n.s. \\
\hline $\begin{array}{l}\text { Klinische } \\
\text { Komplika- } \\
\text { tionen }\end{array}$ & $3,9 \%$ & $0,0 \%$ & 0,056 \\
\hline $\begin{array}{l}\text { Herzinsuffi- } \\
\text { zienz oder } \\
\text { RR-Anstieg } \\
>160 \\
\mathrm{mmHg}\end{array}$ & $19,6 \%$ & $10,4 \%$ & n. s. \\
\hline \multicolumn{4}{|c|}{$\begin{array}{l}\text { Klinische Komplikationen: } 1 \text { Todesfall, } 1 \text { Apoplex, } \\
3 \text { Rehospitalisierungen; } n \text {. s. = nicht signifikant }\end{array}$} \\
\hline
\end{tabular}

Wirkungen verloren, d.h. Symptome einer Herzinsuffizienz und Blutdruckanstiege können auftreten. Signifikant ist allerdings nur der Kreatininanstieg.

Bainey KR et al. Effects of withdrawing vs continuing renin-angiotensin blockers on incidence of acute kidney injury in patients with renal insufficiency undergoing cardiac catheterization (CAPTAIN trial). Am Heart J. 2015;170:110-116.

\section{Kommentar}

Einige teils umfangreiche Beobachtungsstudien und randomisierte Studien haben widersprüchliche Daten zur Wirkung von RAS-Blockern nach der Gabe von Röntgenkontrastmitteln gebracht. In der vorliegenden Studie erreicht der primäre Endpunkt zwar statistische Signifikanz, doch ist der mittlere Anstieg um 0,2 mg/dl sehr gering. Immerhin haben verschiedene Untersuchungen gezeigt, dass Kreatininanstiege nach Herzkatheteruntersuchungen und Revaskularisierungen kardiovaskuläre Komplikationen begünstigen, was trendmäßig auch die vorliegende Studie bestätigt.

Die Autoren argumentieren, dass das vorübergehende Absetzen der RAS-Blocker eine kostenfreie Intervention mit potenziellem Nutzen ist. Andererseits zeigt die Studie auch mögliche Nachteile der Therapieunterbrechung, nämlich vermehrte Symptome einer Herzinsuffizienz und moderate Blutdruckanstiege.

Wer außerhalb von Studien in der Alltagsroutine den grenzwertigen Nutzen sucht, muss auch die Logistik des zeitgerechten Absetzens und der Wiederverordnung der RAS-Blocker beherrschen.

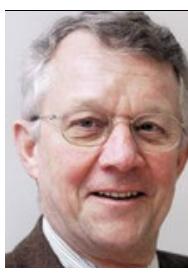

